\title{
On The Homogeneous Quintic Equation with Five Unknowns
}

$$
x^{5}-y^{5}+x y\left(x^{3}-y^{3}\right)=34\left((x+y)\left(z^{2}-w^{2}\right) P^{2}\right.
$$

M.A. Gopalan ${ }^{1}$, S.Vidhyalakshmi ${ }^{2}$, S.Mallika, ${ }^{3}$

.Department of Mathematics, Shrimati Indira Gandhi college,Trichirappalli.620002

\begin{abstract}
The quintic Diophantine equation with five unknowns given by $x^{5}-y^{5}+x y\left(x^{3}-y^{3}\right)=34\left((x+y)\left(z^{2}-w^{2}\right) P^{2} \quad\right.$ is analyzed for its infinitely many non-zero distinct integral solutions. A few interesting relations between the solutions and special numbers namely, centered polygonal numbers, centered pyramidal numbers, jacobsthal numbers, lucas numbers and kynea numbers are presented.
\end{abstract}

Keywords: Quintic equation with five unknowns, Integral solutions, centered polygonal numbers, centered pyramidal numbers.

\section{Introduction}

The theory of Diophantine equations offer a rich variety of fascinating problems.In particular quintic equations homogeneous or non-homogeneous have aroused the interest of numerous mathematicians since antiquity[1,2,3].For illustration, one may refer [4-14],for quintic equations with three ,four and five unknowns. This paper concerns with the problem of determining integral solutions of the non-homogeneous quintic equation with five unknowns given by $x^{5}-y^{5}+x y\left(x^{3}-y^{3}\right)=34\left((x+y)\left(z^{2}-w^{2}\right) P^{2}\right.$. A few relations between the solutions and the special numbers are presented..

\section{Notations}

$t_{m, n}=n\left(1+\frac{(n-1)(m-2)}{2}\right)$.-Polygonal number of rank $\mathrm{n}$ with size $\mathrm{m}$.

$P_{n}^{m}=\left(\frac{n(n+1)}{6}\right)[(m-2) n+(5-m)]$

$P t_{n}=\frac{n(n+1)(n+2)(n+3)}{24}-$ pentatope number of rank $\mathrm{n}$.

$S O_{n}=n\left(2 n^{2}-1\right)$ - Stella octangular number of rank $\mathrm{n}$.

$S_{n}=6 n(n-1)+1_{-}$Star number of rank $\mathrm{n}$.

$\operatorname{Pr}_{n}=n(n+1)$ - Pronic number of rank $\mathrm{n}$.

$J_{n}=\frac{1}{3}\left(2^{n}-(-1)^{n}\right)$-Jacobsthal number of rank $\mathrm{n}$.

$j_{n}=\left(2^{n}+(-1)^{n}\right)$ - Jacobsthal lucas number of rank $\mathrm{n}$.

$K y_{n}=\left(2^{n}+1\right)^{n}-2$ - Kynea number.

$F_{4, m, 3}=\frac{n(n+1)(n+2)(n+3)}{4 !}$-Four dimensional figurative number of rank $\mathrm{n}$ whose generating polygon is a triangle.

$F_{5, m, 3}=\frac{n(n+1)(n+2)(n+3)(n+4)}{5 !}$-Five dimensional figurative number of rank $\mathrm{n}$ whose generating polygon is a triangle.

$C P_{n}^{m}=\frac{m n(n-1)}{2}+1$ - Centered polygonal number of rank $\mathrm{n}$ with size $\mathrm{m}$. 


\section{Method of Analysis}

The Diophantine equation representing the quintic with five unknowns under consideration is

$$
x^{5}-y^{5}+x y\left(x^{3}-y^{3}\right)=34\left((x+y)\left(z^{2}-w^{2}\right) P^{2}\right.
$$

Introducing the transformations

$$
x=u+v, y=u-v, z=u v+1, w=u v-1
$$

in (1), it leads to

$$
u^{2}+v^{2}+=17 p^{2}
$$

which is solved in different ways leading to different solution patterns to (1).

\subsection{Pattern : I}

Assume $p=a^{2}+b^{2}$

write 17 as

$$
17=(1+4 i)(1-4 i)
$$

Substituting (4) and (5) in (3) and employing the method of factorization ,define

$$
u+i v=(1+4 i)(a+i b)^{2}
$$

Equating real and imaginary parts, we get

$$
\begin{aligned}
& u=a^{2}-b^{2}-8 a b \\
& v=4 a^{2}-4 b^{2}+2 a b
\end{aligned}
$$

Thus, in view of (2), the non-zero distinct integral solutions of (1) are given by

$$
\begin{aligned}
& x(a, b)=5 a^{2}-5 b^{2}-6 a b \\
& y(a, b)=-3 a^{2}+3 b^{2}-10 a b \\
& z(a, b)=\left(a^{2}-b^{2}-8 a b\right)\left(4 a^{2}-4 b^{2}+2 a b\right)+1 \\
& w(a, b)=\left(a^{2}-b^{2}-8 a b\right)\left(4 a^{2}-4 b^{2}+2 a b\right)-1 \\
& p(a, b)=a^{2}+b^{2}
\end{aligned}
$$

\subsubsection{Properties}

1) $x(a, a+1)+y(a, a+1)-P(a, a+1)+36 t_{3, a} \equiv 1(\bmod [a+1])$

2) $z(a, 1)+w(a, 1)-96 P t_{a}-48 f_{4, a, 2}+50 S o_{a}+2 t_{114, a} \equiv 0(\bmod a)$

3) $x\left(2^{n}, 1\right)+y\left(2^{n}, 1\right)+P\left(2^{n}, 1\right)-3 K y_{n} \equiv 0(\bmod 2)$

$4\left(x\left(1,2^{n}\right)-y\left(1,2^{n}\right)-P\left(1,2^{n}\right)+j_{2 n}+8 K y_{n}=0\right.$

5) $x(a, 1) P(a, 1)+3 S o_{a}-24 f_{4, a, 7}+42 P_{a}^{4}+28 t_{4, a}+5=0$

6) $x(a(a+1), a)-y(a(a+1), a)-32\left(t_{3, a}\right)^{2}+8 t_{4, a}-8 P_{a}^{5}=0$

7) $z(1, b)+w(1, b)-96 f_{4, b, 2}+42 O H_{b} \equiv 0(\bmod 2)$

\subsection{Pattern: II}

Instead of (5) write 17 as

$$
17=(4+i)(4-i)
$$

For this choice, after performing calculations similar to pattern.I ,the corresponding non-zero integral solutions to (1) are found to be

$$
\begin{aligned}
& x(a, b)=5 a^{2}-5 b^{2}+6 a b \\
& y(a, b)=3 a^{2}-3 b^{2}+10 a b \\
& z(a, b)=\left(4 a^{2}-4 b^{2}-2 a b\right)\left(a^{2}-b^{2}+8 a b\right)+1 \\
& w(a, b)=\left(4 a^{2}-4 b^{2}-2 a b\right)\left(a^{2}-b^{2}+8 a b\right)-1 \\
& P(a, b)=\left(a^{2}+b^{2}\right)
\end{aligned}
$$




\subsection{Pattern: III}

Rewrite (3) as

$$
17 P^{2}-v^{2}=u^{2} * 1
$$

Assume

$$
u=17 a^{2}-b^{2}
$$

Write 1 as

$$
1=[\sqrt{17}+4][\sqrt{17}-4]
$$

Using (8) and (9) in (7) and employing the method of factorization, define

$$
(\sqrt{17} P+v)=(\sqrt{17}+4)(\sqrt{17} a+b)^{2}
$$

Equating rational and irrational parts, we get

$$
\begin{aligned}
& P=17 a^{2}+b^{2}+8 a b \\
& \left.v=68 a^{2}+4 b^{2}+34 a b\right)
\end{aligned}
$$

Substituting the values of $\mathrm{u}$ and $\mathrm{v}$ in (2), the non-zero distinct integral solutions of (1) are as follows.

$$
\begin{aligned}
& x(a, b)=85 a^{2}+3 b^{2}+34 a b, \\
& y(a, b)=-51 a^{2}-5 b^{2}-34 a b \\
& z(a, b)=\left[17 a^{2}-b^{2}\right]\left[68 a^{2}+4 b^{2}+34 a b\right]+1 \\
& w(a, b)=\left[17 a^{2}-b^{2}\right]\left[68 a^{2}+4 b^{2}+34 a b\right]-1 \\
& P(a, b)=17 a^{2}+b^{2}+8 a b
\end{aligned}
$$

\subsubsection{Properties}

1. $3\left[x\left(2^{n}, 1\right)+y\left(2^{n}, 1\right)-102 J_{2 n}\right]$ is a nasty number.

2. $x(1, b+1)-y(1, b+1)+P(1, b+1)-S_{b}-6 t_{3, b} \equiv 43(\bmod 97)$

3. $z(a, 1)-6936 f_{4, a, 5}+4624 P_{a}^{5}+289 S o_{a} \equiv 4(\bmod a)$.

4. $x(a, 1)-P(a, 1)-136 t_{3, a}$ is divisible by 2 .

5. $x\left(1,2^{n}\right)+y\left(1,2^{n}\right)-P\left(1,2^{n}\right)+3 K y_{n}+3 J_{n}+j_{n}=17$

\subsection{Pattern :IV}

Instead of (9), one may write 1 as

$$
1=\frac{[\sqrt{17}+1][\sqrt{17}-1]}{16}
$$

Substituting (8) and (10) in (7) and employing the method of factorization, define

$$
\sqrt{17} P+v=[\sqrt{17} a+b]^{2} \frac{[\sqrt{17}+1]}{4}
$$

Equating rational and irrational parts ,we get

$$
\begin{aligned}
& P=\frac{17 a^{2}+b^{2}+2 a b}{4} \\
& v=\frac{17 a^{2}+b^{2}+34 a b}{4}
\end{aligned}
$$

Since our interest is on finding integral solutions, it is possible to choose $\mathrm{a}$ and $\mathrm{b}$ so that $\mathrm{P}$ and $\mathrm{v}$ are integers.

\subsubsection{Choice: 1}

Let $a=2 A, b=2 B$

Then

$$
\begin{aligned}
& P=17 A^{2}+B^{2}+2 A B \\
& v=17 A^{2}+B^{2}+34 A B
\end{aligned}
$$




$$
u=68 A^{2}-4 B^{2}
$$

Substituting these values in (2) the corresponding integral solutions to (1) are given by,

$$
\begin{aligned}
& x(A, B)=85 A^{2}-3 B^{2}+34 A B \\
& y(A, B)=51 A^{2}-5 B^{2}-34 A B \\
& z(A, B)=\left[68 A^{2}-4 B^{2}\right]\left[17 A^{2}+B^{2}+34 A B\right]+1 \\
& w(A, B)=\left[68 A^{2}-4 B^{2}\right]\left[17 A^{2}+B^{2}+34 A B\right]-1 \\
& P(A, B)=17 A^{2}+B^{2}+2 A B
\end{aligned}
$$

NOTE; Suppose we choose A,B such that $A \succ B \succ 0$ then $u \succ v$.Considering u, $\mathrm{v}$ to be the generators of a Pythagorean triangle, then its area is represented by $x y[z+w]$

\subsubsection{Choice :II}

Let $a=(2 k-1) b$

Then

$$
\begin{aligned}
& P=\left[17 k^{2}-16 k+4\right] b^{2} \\
& v=\left[17 k^{2}+4\right] b^{2} \\
& u=\left[68 k^{2}-68 k+16\right] b^{2}
\end{aligned}
$$

Substituting these values in (2),the non-zero distinct integer solutions of (1) is found to be,

$$
\begin{aligned}
& x=\left[85 k^{2}-68 k+20\right] b^{2} \\
& y=\left[51 k^{2}-68 k+12\right] b^{2} \\
& z=\left[68 k^{2}-68 k+16\right]\left[17 k^{2}+4\right] b^{4}+1 \\
& w=\left[68 k^{2}-68 k+16 k\right]\left[17 k^{2}+4\right] b^{4}-1 \\
& P=\left[17 k^{2}-16 k+4\right] b^{2}
\end{aligned}
$$

\subsubsection{Choice: III}

Let $b=(2 k+1) a$

Then

$$
\begin{aligned}
& P=\left[k^{2}+2 k+5\right] a^{2} \\
& v=\left[k^{2}+18 k+13\right] a^{2} \\
& u=\left[16-4 k^{2}-4 k\right] a^{2}
\end{aligned}
$$

Then the corresponding non-zero distinct integral solutions of (1) are given by,

$$
\begin{aligned}
& x=\left[-3 k^{2}+14 k+29\right] a^{2} \\
& y=\left[-5 k^{2}-22 k+3\right] a^{2} \\
& z=\left[16-4 k^{2}-4 k\right]\left[k^{2}+18 k+13\right] a^{4}+1 \\
& w=\left[16-4 k^{2}-4 k\right]\left[k^{2}+18 k+13\right] a^{4}-1 \\
& P=\left[k^{2}+2 k+5\right] a^{2}
\end{aligned}
$$

.Remark: It is worth mentioning here that the triple $(x, y, z)$ and $(x, y, w)$ obtained from any of the above patterns satisfy respectively the following hyperbolic paraboloids. $x^{2}-y^{2}=4(z-1)$ and $x^{2}-y^{2}=4(w+1)$.

\section{Conclusion}

One may search for other choices of solutions to (1) along with the corresponding properties. 


\section{References}

[1]. L.E.Dickson, History of Theory of Numbers, Vol.11, Chelsea Publishing company, New York (1952).

[2]. L.J.Mordell, Diophantine equations, Academic Press, London(1969).

[3]. Carmichael, R.D.,The theory of numbers and Diophantine Analysis,Dover Publications, New York (1959)

[4]. M.A.Gopalan \& A.Vijayashankar, An Interesting Diophantine problem $x^{3}-y^{3}=2 z^{5}$ Advances in Mathematics, Scientific Developments and Engineering Application, Narosa Publishing House, Pp 1-6, 2010.

[5]. M.A.Gopalan \& A.Vijayashankar, Integral solutions of ternary quintic Diophantine equation $x^{2}+(2 k+1) y^{2}=z^{5}$,International Journal of Mathematical Sciences 19(1-2), 165-169,(jan-june 2010)

[6]. M.A.Gopalan,G.Sumathi \& S.Vidhyalakshmi, Integral solutions of non-homogeneous Ternary quintic equation in terms of pells sequence $x^{3}+y^{3}+x y(x+y)=2 z^{5}$ Journal of Applied Mathematical Sciences, vol.6,No.1,59-62,April.2013.

[7]. M.A.Gopalan,G.Sumathi and S.Vidhyalakshmi "'Integral solutions of non- homogeneous quintic equation with three unknowns $x^{2}+y^{2}-x y+x+y+1=\left(k^{2}+3\right)^{n} z^{5}$ ''International J. of innovative Research in science engineering and tech.Vol.2,issue.4,920-925,April.2013.

[8]. S.Vidhyalakshmi, K.Lakshmi and M.A.Gopalan, Observations on the homogeneous quintic equation with four unknowns $x^{5}-y^{5}=2 z^{5}+5(x+y)\left(x^{2}-y^{2}\right) w^{2}$,accepted for Publication in International Journal of Multidisciplinary Research Academy (IJMRA).

[9]. M.A.Gopalan,S.Vidhyalakshmi and A.Kavitha On the quintic equation with four unknowns $x^{4}-y^{4}=2\left(k^{2}+t^{2}\right) z^{4} w$ accepted for publication in Bessel J. of Mathematics10.M.A.Gopalan,G.Sumathi and S.Vidhyalakshmi,' Integral solutions of the $\begin{array}{llll}\text { non--homogeneous quintic } & \text { equation } & \text { four } & \text { unknowns }\end{array}$ $x^{5}-y^{5}+\left(x^{4}+y^{4}\right) z+52 w^{4} z=4 z\left(1+7 w^{2}\right)$ '’Bessel J. of Mathematics,3(1),175-180,2003.

[10]. M.A.Gopalan \& A.Vijayashankar, Integral solutions of non-homogeneous quintic equation with five unknowns , Bessel J.Math.,1(1),23-30,2011.

[11]. M.A.Gopalan \& A.Vijayashankar, solutions of quintic equation with fiveunknowns $x^{4}-y^{4}=2\left(z^{2}-w^{2}\right) P^{3}$, Accepted for Publication in International Review of Pure and Applied Mathematics.

[12]. M.A.Gopalan, G. Sumathi \& S.Vidhyalakshmi," On the non-homogenous quintic equation with five unknowns $x^{3}+y^{3}=z^{3}+w^{3}+6 T^{5}$ International Journal of Management, IT and Engineers.Vol.3,Issue.4 501-506,April.2013

[13]. M.A.Gopalan,S.Mallika,and S.Vidhyalakshmi,"'On the non-homogeneous quintic equation with five unknowns $x^{4}-y^{4}=2\left(k^{2}+s^{2}\right)\left(z^{2}-w^{2}\right) p^{3}$ "'International Journal of Innovative Research In Science, Engineering and Technology,Vol.2.Issue 4 1216-1221,,April.2013. 